\title{
Efeitos toxicológicos e ineficiência in vitro de deltametrina sobre larvas de Rhipicephalus sanguineus, deGoiânia, Goiás, Brasil
}

\author{
Toxicological effects and in vitro inefficacy of deltamethrin on larvae of \\ Rhipicephalussanguineusfrom Goiânia, Goiás, Brazil
}

\begin{abstract}
Fernando de Freitas Fernandes ${ }^{1}$, Edméia de Paula e Souza Freitas ${ }^{2}$, José Rubens Veríssimo da Silva ${ }^{2}$,
\end{abstract} Oyama Rodrigues da Silva ${ }^{2}$ e Ionizete Garcia da Silva ${ }^{1}$

\begin{abstract}
Resumo Observou-se que formulações de deltametrina, desenvolvidas para desinsetizar ambientes infestados ou para combate de artrópodes parasitas de bovinos e equinos, têm sido freqüentemente utilizadas por cinofilistas para combater carrapatos em seus cães, sem critério científico em relação às dosagens e formas de aplicação, nos canis e ambientes onde vivem. Com a finalidade de avaliar a atividade deste produto sobre Rhipicephalus sanguineus, realizaram-se bioensaios com larvas a $27 \pm 1^{\circ} \mathrm{C}, U R>80 \%$ e fotofase de $12 \mathrm{~h}$. $\mathrm{A}$ mortalidade $e$ os efeitos toxicológicos foram observados durante $30 \mathrm{~h}$, a cada $6 \mathrm{~h}$. Constataram-se efeitos toxicológicos importantes nas larvas, tais como incoordenação, Knock-down e paralisia seguida por morte, todavia a capacidade letal do produto após 30h foi baixa, com mortalidade média de 34\%, 40,2\%, 46\% e $57,6 \%$, respectivamente, para as dosagens de $0,5 \mathrm{ml}, 1 \mathrm{ml}, 2 \mathrm{ml}, 3 \mathrm{ml}$. Não houve mortalidade no grupo controle. Os resultados obtidos contra-indicam o uso da deltametrina nas dosagens testadas como medida eletiva para controle de $\mathrm{R}$. sanguineus.
\end{abstract}

Palavras-chaves: Rhipicephalus sanguineus. Ixodidae. Controle químico. Deltametrina. Cães.

\begin{abstract}
It has been observed that formulations of deltamethrin developed for disinsectization of infested places and control of parasitic arthropods of bovines and equines have frequently been used by breeders of dogs to control ticks in their animals and kennels, but without due consideration of the correct dosage or means of application. With the purpose of evaluating the efficacy of this product on Rhipicephalus sanguineus, bioassays were realized with larvae at $27 \pm 1^{\circ} \mathrm{C}, U R>80 \%$ and light period of 12 hours. The mortality and toxicological effects were observed for $30 \mathrm{~h}$, at $6 \mathrm{~h}$ intervals. Important toxicological effects were observed on the larvae, such as lack of co-ordination, knock-down and paralysis followed by death, although the lethal capacity of the product after $30 \mathrm{~h}$ was low, with mean mortality of only $34 \%, 40.2 \%, 46 \%$ and $57.6 \%$ respectively, for the $0.5 \mathrm{ml} / \mathrm{l} ; 1 \mathrm{ml} / \mathrm{l} ; 2 \mathrm{ml} / \mathrm{l} / ;$ and $3 \mathrm{ml} / \mathrm{l}$ dosages. There was no mortality in the control group. The results obtained did not indicate the use of this formulation, in the tested dosages, as a method of choice for R. sanguineus control.
\end{abstract}

Key-words: Rhipicephalus sanguineus. Ixodidae. Chemical control. Deltamethrin. Dogs.

Rhipicephalus sanguineus (Latreille, 1806) (Acari, Ixodidae) é um carrapato heteroxeno, cosmopolita, que parasita principalmente o cão doméstico e, secundariamente, outros mamíferos, aves e répteis ${ }^{17}$. É o principal vetor biológico e reservatório da Ehrlichia canis, podendo igualmente transmitir Babesia canis, B. caballi e $B$. equi ${ }^{25}$ e, em $18,8 \%$ desses carrapatos coletados em cães domésticos, do centro e norte do Mississipi e de Chicago, EUA, Rickettsia do grupo etiológico da febre maculosa foi neles diagnosticada 67272836 .
Recentemente, foi comprovado que $R$. sanguineus, principalmente através de suas formas imaturas, alimenta-se em humanos mais freqüentemente do que antes se supunha ${ }^{23}$. Assim, por se desenvolver em ambientes sinantrópicos, de várias cidades do Brasil, onde ocorre em altas densidade e prevalência, este carrapato poderá vir a causar aumento na incidência de erliquiose, babesiose e febre maculosa, como antropozoonoses emergentes ${ }^{6} 2325$. Pela falta de padrões clínicos característicos que as separem de outras doenças com

${ }^{1}$ Setor de Entomologia do Departamento de Microbiologia, Imunologia, Parasitologia e Patologia do Instituto de Patologia Tropical e Saúde Pública da Universidade Federal de Goiás, ²Estagiários em Iniciação Científica.Goiânia, GO.

Endereço para correspondência: Prof. Fernando de Freitas Fernandes. Setor de Entomologia/DMIPP/IPTSP/Universidade Federal de Goiás, Caixa Postal: 131, 74605-050 Goiânia, GO, Brasil.

Fax: 5562 202-3066

e-mail: ffreitas@iptsp.ufg.br

Recebido para publicação em 26/6/2000 
patognomonias semelhantes e mais freqüentes no homem, tais zoonoses muitas vezes são diagnosticadas tardiamente, levando o paciente à morte 222440 . Apesar disto, no Brasil poucos estudos foram realizados com este vetor ${ }^{8} 1213151635$.

Os acaricidas químicos têm sido a principal forma de combate aos carrapatos, todavia, a resistência de $R$. sanguineus aos mesmos ${ }^{32132}$ tem sido diagnosticada em certos países. No Brasil, estudos para o monitoramento da suscetibilidade do $R$. sanguineus aos acaricidas, bem como para sua utilização correta, tornam-se necessários e urgentes, uma vez que diversos deles estão sendo administrados, indiscriminadamente sob formas e dosagens variadas, em cães e ambientes infestados, gerando ineficiência acaricida ${ }^{20}$, prejuízos econômicos aos criadores, intoxicações aos animais e ao homem ${ }^{121516}$.
Provavelmente, o uso de formulações de deltametrina, desenvolvidas para controle de artrópodes parasitos de bovinos e eqüinos, e para desinsetização de ambientes infestados, comercializadas indevidamente para a descarrapatização de cães, canis e demais ambientes infestados, inclusive domicílios, estaria levando ao desenvolvimento de cepas resistentes de $R$. sanguineus. Com o objetivo de subsidiar o controle de $R$. sanguineus e orientar veterinários e cinofilistas, a atividade larvicida e os efeitos toxicológicos deste piretróide, em diferentes dosagens, foram aqui investigados. Outros fatores como a foto-termoestabilidade da deltametrina, a sua baixa toxicidade para mamíferos e alta para insetos, além de resultados anteriores obtidos com este piretróide para o controle de artrópodes de importância agrícola e médico-veterinária9 ${ }^{111431}$, foram também considerados.

\section{MATERIAL E MÉTODOS}

Amostras de teleóginas de $R$. sanguineus foram coletadas de canis infestados, em cinco residências de cinco bairros da cidade de Goiânia, Goiás, escalando paredes, muros, madeiramento do telhado e no piso, ou se escondendo em frestas para realizarem a oviposição (Figura 1). De cada canil, 20 teleóginas foram coletadas e depois levadas ao laboratório para a obtenção de larvas. Uma cadela jovem da raça dobermann, livre de resíduos acaricidas, foi posteriormente infestada artificialmente em um canil com um pool de larvas, originado a partir das oviposturas que apresentaram os melhores índices de eclodibilidade, conforme Amaral ${ }^{1}$. Durante o verão, quando um maior número de ixodídeos foi encontrado

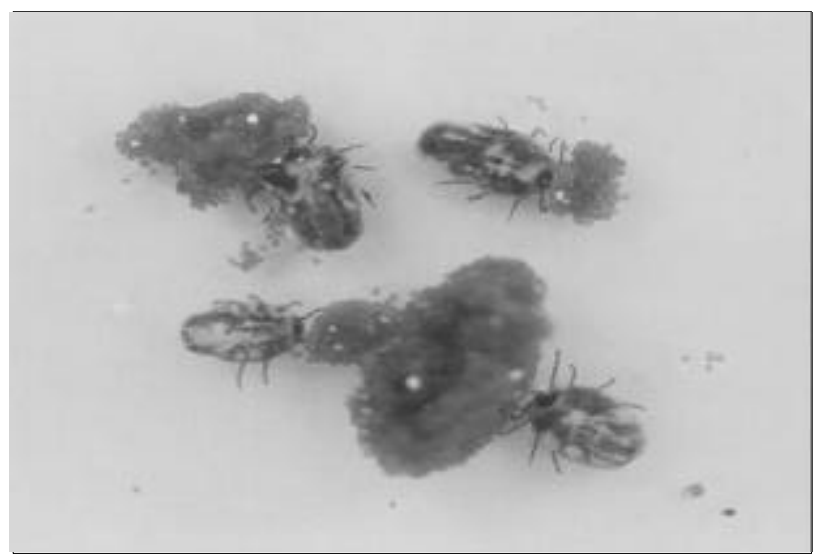

Figura 1 - Teleóginas de Rhipicephalus sanguineus ovipondo. no canil experimental, 20 fêmeas aptas à reprodução, repletas de sangue e desprendidas naturalmente, foram colhidas, acondicionadas em tubos de polietileno e transportadas ao laboratório ${ }^{12}$. Os ixodídeos foram mantidos em B.O.D. a $27 \pm 1^{\circ} \mathrm{C}$, UR $>80 \%{ }^{12}$ e fotoperíodo de 12 horas $^{9}$. Com um estereoscópio verificou-se a ausência de mutilações ou malformações nas teleóginas. Estas foram individualizadas em tubos de polietileno para realizarem a oviposição. Visando a obtenção de larvas com idade uniforme, as oviposturas eram agrupadas diariamente em um único dispositivo ${ }^{10}$, constituído por placa de petri de polietileno $(9 \times 1,4 \mathrm{~cm})$ e coberto com tecido sintético do tipo organza e parafina (Figura 2).

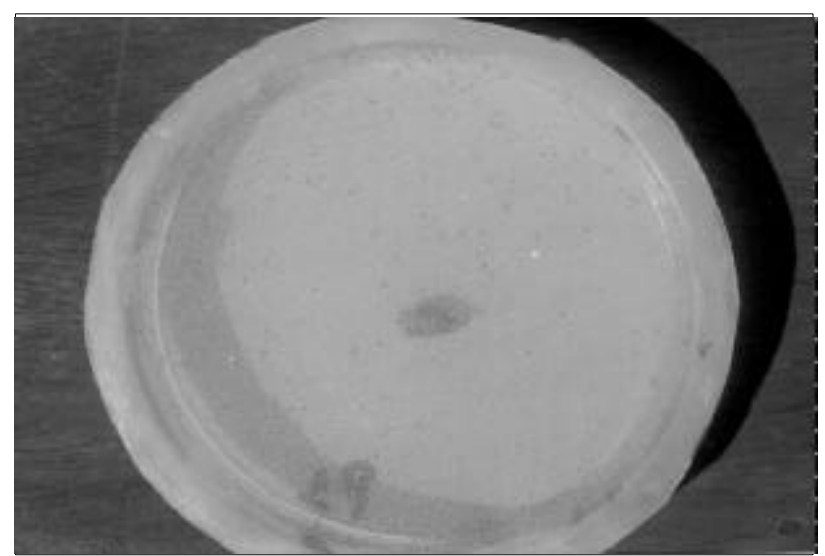

Figura 2 - Eclosão de larvas de Rhipicephalus sanguineus no interior do dispositivo para manuntençáo de colônias de ixodideos em estudos do ciclo biológico. 
Entre as oviposturas com melhor eclodibilidade (> que $90 \%$ ) as larvas com 14 a 21 dias $^{18}$ eram retiradas para os bioensaios, frente a um produto comercial composto por $25 \mathrm{~g}$ de deltametrina base em $1.000 \mathrm{ml}$ de veículo q.s.p.. O produto foi testado naquelas dosagens recomendadas pelo fabricante para pulverizações: bovinos $(1 \mathrm{ml} / \mathrm{l})$, equinos $(2 \mathrm{ml} / \mathrm{l})$, bem como em dosagens superior $(3 \mathrm{ml} / \mathrm{l})$ e inferior $(0,5 \mathrm{ml} / \mathrm{l})$, visando selecionar aquela reduzida e efetiva contra $R$. sanguineus, objetivando assim minimizar os custos, riscos com intoxicação e impacto ambiental. Os volumes calculados para cada dosagem foram pipetados e diluídos em água destilada. Estas soluções foram homogeneizadas por agitação e colocadas em placas de petri, sobre um fundo branco, para melhor visualização das larvas durante a imersão. O dispositivo ${ }^{10}$ selecionado com as larvas foi colocado sobre um becker de $500 \mathrm{ml}$, disposto invertido no centro de uma forma de alumínio ${ }^{12}$ contendo água. Através de uma pequena abertura na margem do dispositivo ${ }^{10}$ as larvas ao saírem voluntariamente ou por estímulo (calor e $\mathrm{CO}_{2}$ da expiração do pesquisador) pelo orifício, eram colhidas aos poucos e colocadas no centro de uma circunferência de $7 \mathrm{~cm}$ de raio, desenhada sobre uma folha de papel, fixada

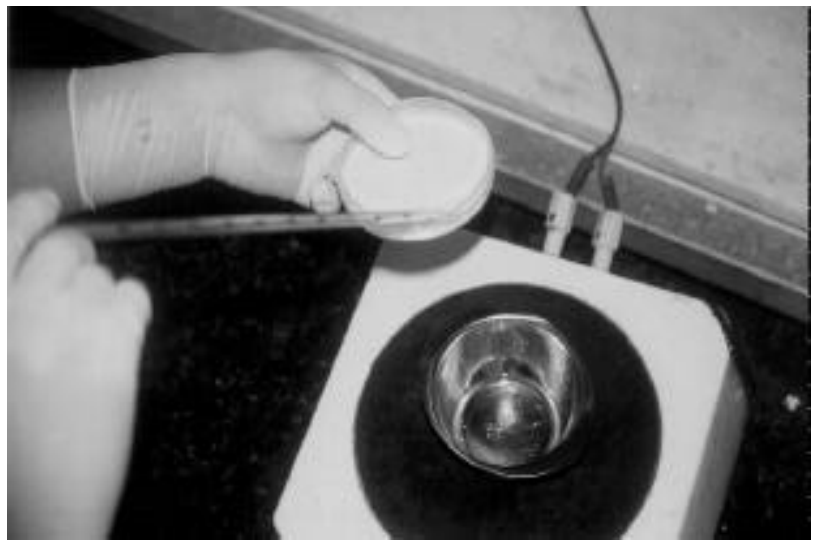

Figura 3A - Dispositivo de contenção de ixodídeos em ensaios toxicológicos. Fechamento com parafina.

acaricida, as larvas eram observadas ao estereoscópio uma hora após a exposição ao acaricida e a cada seis horas, durante $30 \mathrm{~h}$, com a mortalidade sendo expressa em média percentual. Em caso de mortalidade entre $0 \%$, e $5 \%$ no grupo controle seriam elas corrigidas pela fórmula à bancada. Apenas as larvas com boa capacidade locomotora e que rapidamente ultrapassavam o círculo, eram aproveitadas para os testes.

Com um pincel umedecido na solução acaricida a ser testada, as larvas foram içadas e imediatamente imersas na mesma solução, durante 2 minutos. Em seguida, eram colocadas, afastadas uma das outras para melhor visualização, sobre o papel filtro de um dispositivo para exposição de larvas em ensaios com acaricidas desenvolvido por Fernandes ${ }^{12}$ (Figura 3). O papel filtro foi colocado internamente à tampa da placa de petri. A base desta funcionou como tampa do dispositivo, por onde as larvas eram observadas. 0 dispositivo foi vedado colocando-se entre suas bordas parafina em fusão, retirada de uma cuba inoxidável com uma pipeta graduada, ambas mantidas sobre uma manta térmica (Figura 3). Para cada dosagem eram utilizados 10 dispositivos, com pelo menos 20 larvas em cada um deles, identificados e separados sobre uma bancada para observação. O grupo controle teve seleção e o manejo iguais aos do grupo teste, exceto pela imersão em água destilada ${ }^{12}$.

Com a finalidade de verificar os efeitos toxicológicos e a mortalidade causados pela interação ácaro-

\section{RESULTADOS E DISCUSSÃO}

O desenvolvimento do $R$. sanguineus foi obtido satisfatoriamente no canil experimental como em laboratório, com as oviposturas apresentando 80 a

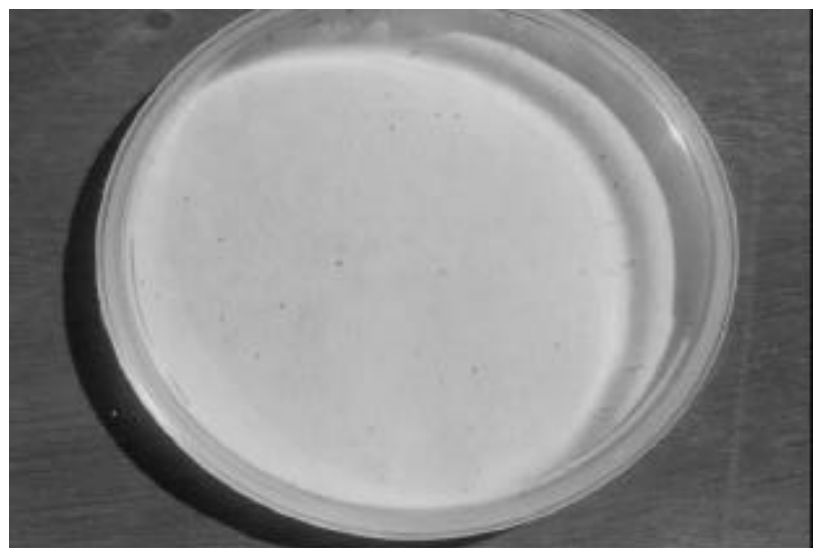

Figura 3B - Observações de larvas de Rhipicephalus sanguineus em seu interior.

de Abbot e; quando acima de $5 \%$, os bioensaios seriam anulados e repetidos ${ }^{18}{ }^{42}$. Aplicou-se teste de qui-quadrado $\left(\mathrm{X}^{2}\right)$ ao nível de $5 \%$ de significância ${ }^{34}$ para verificar a influência da dosagem na eficiência larvicida.

$100 \%$ de eclodibilidade. Conforme Tabelas 1 e 2 e Figura 4, a mortalidade média do $R$. sanguineus observada na $24^{\mathrm{a}} \mathrm{h}$ (padrão $\mathrm{WHO}^{42}$ e $\mathrm{FAO}^{18}$ ) foi de 
Tabela 1 - Mortalidade de larvas de Rhipicephalus sanguineus, por ação da deltametrina em diferentes dosagens, observada a cada 6h por $30 h$.

\begin{tabular}{|c|c|c|c|c|c|c|c|c|}
\hline \multirow{2}{*}{$\begin{array}{l}\text { Dosagem } \\
(\mathrm{ml} / \mathrm{l})\end{array}$} & \multirow{2}{*}{$\begin{array}{l}\mathrm{N}^{\circ} \text { de } \\
\text { larvas }\end{array}$} & \multirow{2}{*}{$\begin{array}{l}\text { Média } \\
\text { L/D }\end{array}$} & \multicolumn{6}{|c|}{ Mortalidade média (\%) / Erro Padrão } \\
\hline & & & $1 \mathrm{~h}$ & $6 \mathrm{~h}$ & $12 \mathrm{~h}$ & $18 \mathrm{~h}$ & $24 \mathrm{~h}$ & $30 \mathrm{~h}$ \\
\hline Controle & 235 & 23,5 & 0 & 0 & 0 & 0 & 0 & 0 \\
\hline 0,5 & 348 & 34,8 & 0 & 0 & $10,6 \pm 0,88$ & $22,1 \pm 0,61$ & $32,7 \pm 0,88$ & $34,0 \pm 0,71$ \\
\hline 1,0 & 313 & 31,3 & 0 & $9,6 \pm 0,25$ & $15,0 \pm 0,73$ & $30,0 \pm 1,52$ & $37,4 \pm 1,22$ & $40,2 \pm 1,16$ \\
\hline 2,0 & 309 & 30,9 & 0 & $10,0 \pm 2,33$ & $20,1 \pm 0,46$ & $31,1 \pm 0,94$ & $40,1 \pm 1,05$ & $46,0 \pm 0,86$ \\
\hline 3,0 & 231 & 23,1 & 0 & $13,8 \pm 0,53$ & $32,0 \pm 0,56$ & $45,4 \pm 0,34$ & $51,5 \pm 0,43$ & $57,6 \pm 0,47$ \\
\hline
\end{tabular}

Média L/D: número médio de larvas/dispositivo.

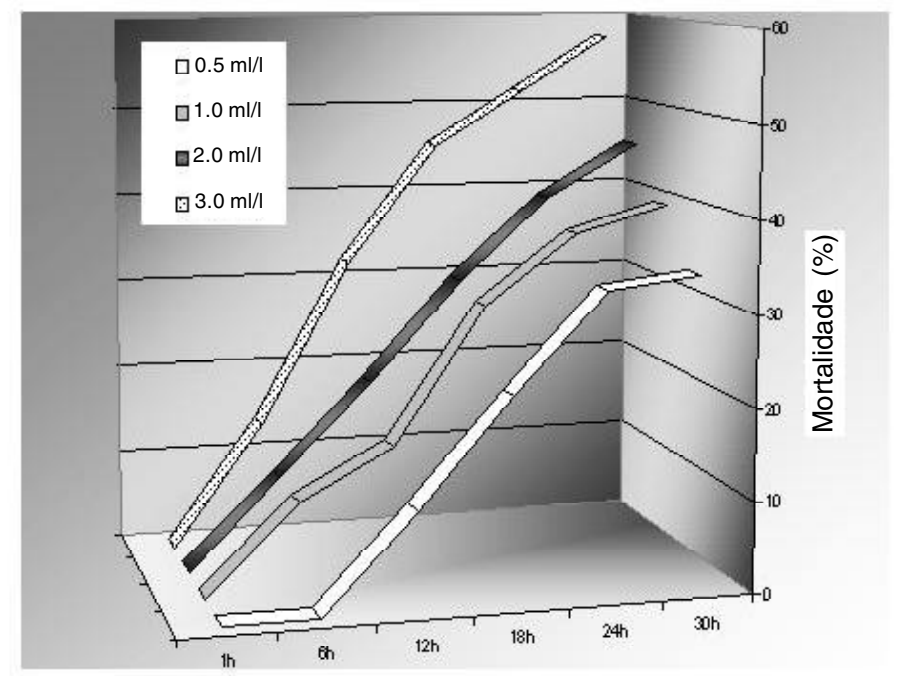

Figura 4 - Ação da deltametrina em diferentes dosagens e horários de observação sobre larvas de Rhipicephalus sanguineus.

$32,7 \%, 37,4 \%, 40,1 \%$ e $51,5 \%$ para as dosagens de $0,5 \mathrm{ml} / \mathrm{l}, 1 \mathrm{ml} / \mathrm{l}, 2 \mathrm{ml} / \mathrm{l} \mathrm{e} 3 \mathrm{ml} / \mathrm{l}$, respectivamente. Na mesma seqüência de dosagens, após $30 \mathrm{~h}$ a mortalidade média foi de $34 \%, 40,2 \%, 46 \%, 57,6 \%$. Diferenças significativas $(p<0,05)$ foram observadas entre as mortalidades médias nas dosagens de $0,5 \mathrm{ml} / /$ e $1 \mathrm{ml} / \mathrm{l}$ e $2 \mathrm{ml} / \mathrm{l}$ e $3 \mathrm{ml} / \mathrm{l}$, observadas após 30h.

De acordo com a Organização Mundial de Saúde ${ }^{42}$, a mortalidade média por inseticidas igual ou superior a $80 \%$ configura o status suscetível ao vetor. No Brasil, para a obtenção de registro de acaricidas em espécies animais junto ao Ministério da Agricultura, devem eles ser capazes de matar 95\% dos ixodídeos na dosagem recomendada ${ }^{30}$. Conseqüentemente, além da baixa eficiência larvicida observada, os resultados confirmaram o desenvolvimento de resistência da cepa de $R$. sanguineus face a todas as dosagens ora testadas.

Em outros países, a resistência de $R$. sanguineus a organofosforados e carbamatos ${ }^{32}$, organofosforados e arsenicais ${ }^{21}$ e organofosforados e organoclorados ${ }^{3}$, tem sido notificada. No Brasil, resistência de Boophilus microplus à deltametrina foi recentemente noticiada em Goiânia, Goiás ${ }^{11}$ e para $R$. sanguineus, os primeiros relatos de ineficiência carrapaticida ocorreram a partir de $1997^{1215}$. Estes dados, aliados aos resultados do presente trabalho, fortalecem a hipótese levantada.

Franc e Cadiergues ${ }^{19}$, divergindo dos resultados supracitados, relataram eficácia ao nível de 95\% contra $R$. sanguineus após $48 \mathrm{~h}$ em tratamentos com xampu de deltametrina, ainda que empregando metodologia diferente das autorizadas para o diagnóstico de resistência a acaricidas, as quais utilizam larvas em jejum e in vitro ${ }^{18}{ }^{39}$. Por outro lado, nem sempre o desprendimento de ixodídeos dos cães pela aplicação de acaricidas é fato comprobatório de eficiência carrapaticida, uma vez que parte desses carrapatos assim desprendidos podem vir a se recuperar ${ }^{12} 1524$. Acresce que a propagação de um determinado alelo resistente na população de carrapatos pode ser devida à sobrevivência preferencial dos indivíduos resistentes, após exposição ao acaricida ${ }^{20}$.

Uma vez que em metodologias usuais, desenvolvidas para o estudo da suscetibilidade de ixodídeos aos carrapaticidas, a observação da mortalidade é geralmente realizada ao final da experimentação (24h), quando são abertos os envelopes de contenção de larvas, constituídos de papel filtro $^{18}{ }^{38} 39$, ou semelhantes a saquinhos de chá ${ }^{21}$, os efeitos toxicológicos provocados nas larvas, bem como 
Tabela 2- Atividade da deltametrina sobre larvas de Rhipicephalus sanguineus, em diferentes dosagens, observadas durante 30 horas.

\begin{tabular}{|c|c|c|c|c|c|c|c|}
\hline \multirow[t]{2}{*}{$\begin{array}{l}\text { Dispositivos } \\
\text { dosagem (ml/l) }\end{array}$} & \multirow[t]{2}{*}{ № de larvas } & \multicolumn{5}{|c|}{ Mortalidade } & \multirow[b]{2}{*}{$30 \mathrm{~h}$} \\
\hline & & $01 \mathrm{~h}$ & $06 \mathrm{~h}$ & $12 \mathrm{~h}$ & $18 \mathrm{~h}$ & $24 \mathrm{~h}$ & \\
\hline$\overline{0,5}$ & 34 & 0 & 0 & 4 & 7 & 10 & 11 \\
\hline 0,5 & 33 & 0 & 0 & 3 & 7 & 8 & 9 \\
\hline 0,5 & 38 & 0 & 0 & 5 & 12 & 13 & $11^{\mathrm{KD}}$ \\
\hline 0,5 & 34 & 0 & 0 & 6 & 8 & 11 & 11 \\
\hline 0,5 & 36 & 0 & 0 & 3 & 6 & 15 & 15 \\
\hline 0,5 & 31 & 0 & 0 & 10 & $9^{* \mathrm{KD}}$ & 13 & 13 \\
\hline 0,5 & 39 & 0 & 0 & 2 & 7 & 8 & 10 \\
\hline 0,5 & 34 & 0 & 0 & 0 & 7 & 9 & 10 \\
\hline 0,5 & 32 & 0 & 0 & 2 & 5 & 16 & 16 \\
\hline 0,5 & 37 & 0 & 0 & 2 & 9 & 11 & 12 \\
\hline 1,0 & 31 & 0 & 2 & 4 & 10 & 12 & 14 \\
\hline 1,0 & 33 & 0 & 4 & 6 & 9 & 10 & 11 \\
\hline 1,0 & 29 & 0 & 2 & 3 & 6 & 9 & 10 \\
\hline 1,0 & 33 & 0 & 4 & 7 & 10 & 14 & 18 \\
\hline 1,0 & 25 & 0 & 3 & 3 & 8 & 8 & 9 \\
\hline 1,0 & 43 & 0 & 3 & 8 & 17 & 18 & 18 \\
\hline 1,0 & 33 & 0 & 4 & 8 & 18 & 18 & $16^{* \mathrm{KD}}$ \\
\hline 1,0 & 22 & 0 & 3 & $2^{* K D}$ & 5 & 7 & 8 \\
\hline 1,0 & 31 & 0 & 2 & 3 & 3 & 10 & 10 \\
\hline 1,0 & 33 & 0 & 3 & 3 & 8 & 11 & 12 \\
\hline 2,0 & 34 & 0 & 4 & 6 & 9 & 12 & 15 \\
\hline 2,0 & 28 & 0 & 2 & 5 & 9 & 13 & 13 \\
\hline 2,0 & 37 & 0 & 4 & 9 & 15 & 18 & $17^{\mathrm{KD}}$ \\
\hline 2,0 & 31 & 0 & 3 & 6 & 15 & 18 & 18 \\
\hline 2,0 & 20 & 0 & 2 & 4 & 7 & 8 & 10 \\
\hline 2,0 & 28 & 0 & 3 & 5 & 8 & 12 & 14 \\
\hline 2,0 & 41 & 0 & 4 & 8 & 10 & 13 & 18 \\
\hline 2,0 & 30 & 0 & 3 & 6 & 8 & 10 & 12 \\
\hline 2,0 & 29 & 0 & 3 & 7 & 8 & 10 & 13 \\
\hline 2,0 & 31 & 0 & 3 & 6 & 7 & 10 & 12 \\
\hline 3,0 & 27 & 0 & 2 & 8 & 12 & 13 & 16 \\
\hline 3,0 & 25 & 0 & 4 & 4 & 10 & 14 & 14 \\
\hline 3,0 & 22 & 0 & 5 & 7 & 10 & 11 & 13 \\
\hline 3,0 & 20 & 0 & 1 & 6 & 12 & $11^{\mathrm{K} \mathrm{KD}}$ & 11 \\
\hline 3,0 & 20 & 0 & 4 & 6 & 9 & 10 & 12 \\
\hline 3,0 & 24 & 0 & 3 & 7 & 9 & 11 & 13 \\
\hline 3,0 & 20 & 0 & 1 & 10 & 10 & 11 & 13 \\
\hline 3,0 & 20 & 0 & 2 & 9 & 11 & 12 & 12 \\
\hline 3,0 & 26 & 0 & 4 & 8 & 11 & 14 & 15 \\
\hline 3,0 & 27 & 0 & 6 & 9 & 11 & 12 & 14 \\
\hline
\end{tabular}

${ }^{*} \mathrm{KD}$ : knock-down.

os tempos letais, que são importantes aspectos na interação ácaro-acaricida, não são observados. Por tais razões, ao contrário de outros estudos que utilizam envelopes opacos, o dispositivo de contenção de larvas proposto por Fernandes ${ }^{12}$ foi aqui empregado, e proporcionou uma perfeita visualização das larvas, sem a necessidade de ser aberto interrompendo a experimentação. Sendo ele constituído por materiais descartáveis de baixo custo e acessíveis no mercado, a sua utilização demonstrou praticabilidade pela ausência de toxicidade às larvas, não permitindo mortalidade no grupo controle. Tal dispositivo, testado neste trabalho para a contenção de 1.436 larvas, além de não permitir o escape das larvas, e pelo fato de ser manuseado lacrado, elimina o risco de se confundir movimento espontâneo de larvas com aqueles provocados em larvas mortas pela respiração do pesquisador que as observa.

O perfil toxicológico do piretróide, observado ao estereoscópio evidenciou seqüencialmente: maior 
excitação das larvas no início, seguida por movimentação repetitiva, diminuição progressiva da capacidade locomotora, tremores, prostração, movimentação apenas das patas e palpos, estendimento das patas levando ao desprendimento, paralisia evoluindo para a recuperação dos movimentos (efeito knock-down) ou para a morte (efeito killing) ${ }^{24}$. Algumas larvas, ainda vivas, apresentaram proliferação de líquido e gases em seu interior que, inicialmente dilatando pequenas áreas da cutícula, evoluíram posteriormente para o comprometimento progressivo de todo o idiossoma, culminando com morte. O efeito knock-down, característico da ação de piretróides sobre artrópodes e confundível com morte por inibição reversível dos movimentos ${ }^{24}$, já havia sido observado por outros autores, em culicíneos na 36 ${ }^{\text {a }} \mathrm{h}$ de exposição à deltametrina ${ }^{937}$, embutindo uma falsa mortalidade de até $16,5 \%$. A ocorrência deste efeito toxicológico suscita a necessidade de observação da mortalidade por um tempo maior que $24 \mathrm{~h}$, como constatado para larvas de $R$. sanguineus submetidas à cipermetrina ${ }^{16}$.

Cumpre salientar que os resultados ora obtidos são específicos para a cepa da microrregião estudada. Na maioria das vezes, algumas reclamações de ineficiência carrapaticida, quando verificadas por médicos veterinários competentes, devem-se a problemas relacionados com diluição, homogeneização, validade, armazenamento e administração do acaricida, etc. Desta forma, testes precisos devem ser realizados por pesquisadores, médicos veterinários especialistas em controle químico de vetores, em cada região onde hajam suspeitas de ineficácia carrapaticida, com suas respectivas cepas $^{20}$. Em plena virada do milênio é fundamental que o uso produtos químicos se restrinja ao estritamente necessário, visando reduzir o impacto ambiental, causado pelo acúmulo destas drogas no ambiente e cadeias alimentares. Ainda que métodos físicos de combate, como a utilização de lança-chamas de uso doméstico (vassoura-de-fogo) sobre superfícies infestadas possam ser empregados, outras perspectivas para controle deveriam ser incentivadas tais como: vacina anticarrapato ${ }^{29} 33$, acaricidas fitossanitários $^{40}$ e à base de microorganismos entomopatogênicos ${ }^{4} 42$ e armadilhas com feromônios ou outros atraentes semioquímicos ${ }^{22}$.

\section{REFERÊNCIAS BIBLIOGRÁFICAS}

1. Amaral NK. Guidelines for the evaluation against the cattle tick Boophilus microplus (Canestrini, 1887) (Acari: Ixodidae). Revista Brasileira de Parasitologia Veterinária 2:144-151, 1993.

2. Andereg PI, Passos LMF. Erliquiose canina-revisão. Clínica Veterinária 12:31-38, 1999.

3. Belot J, Mishra G. Toxicité de huit produits acaricides différents sur le tiques de l'espèce Rhipicephalus sanguineus. Recueil de Médicine Vétérinaire 155: 869-871, 1979.

4. Bittencourt VREP, Peralva SLFS, Viegas EC, Alves SB. Avaliação do efeito do contato de Beuveria bassiana (Bals.) Vuill com ovos e larvas de B. microplus (Canestrini, 1887) (Acari: Ixodidae). Revista Brasileira de Parasitologia Veterinária 5:81-84, 1996.

5. Breitschwerdt EB, Hegarty BC, Hancock SI. Sequential evaluation of dogs naturally infected with Ehrlichia canis, E. chaffeensis, E. equi, E. ewingii or Bartonella vinsolnii. Journal of Clinical Microbiology 36:2645-2651, 1998.

6. Burgdorfer W, Sexton DJ, Gerloff RK, Anacker RL, Philip RN Thomas LA. Rhipicephalus sanguineus: vector of a new spotted fever group rickettsia in the United States. Infection and Immunity 12:205-210, 1975.

7. Castellà Espuny J, Gutiérrez Galindo J, Estrada Peña A, Muñoz Lopez E. Actividad ovicida de diversos compuestos acaricidas sobre puestas de la garrapata del perro Rhipicephalus sanguineus, Latreille (Acari: Ixodidae). Medicina Veterinária 11:396-401, 1994.

8. Famadas KM, Mota CS. Órgão de Haller: caracterização morfológica e estruturas setiformes anexas presentes no tarso I da larva de Rhipicephalus sanguineus (Acari: Ixodidae). In: Anais do XVI Congresso Brasileiro de Entomologia, Salvador p. 42, 1997.

9. Fernandes FF. Ação da deltametrina na formulação de ultrabaixo-volume em diferentes horários e dosagens, sobre o Aedes (Stegomyia) aegypti (Linnaeus, 1762) (Diptera,
Culicidae). Tese de Mestrado, Universidade Federal de Goiás, GO, 1996.

10. Fernandes FF. Dispositivo experimental para manutenção de colônias de ixodídeos visando ao estudo dos parâmetros biológicos da fase de vida livre do ciclo evolutivo. Revista Goiana de Medicina 42:43-48, 1997.

11. Fernandes FF. Estudo dos efeitos toxicológicos e eficiência larvicida de piretróides sobre Boophilus microplus (Canestrini, 1887). In: Resumos do XVI Congresso Brasileiro de Parasitologia, Poços de Caldas, p.59, 1999.

12. Fernandes FF. In vitro activitty of permethrin, cipermethrin and deltamethrin on larvae of Rhipicephalus sanguineus (Latreille, 1806) (Acari, Ixodidae). Arquivo Brasileiro de Medicina Veterinária e Zootecnia 52: 621-626, 2000.

13. Fernandes FF, Freitas EPS. Avaliação da atividade de fenthion em formulação epicutânea no controle de Rhipicephalus sanguineus (Latreille, 1806) (Acari, Ixodidae) em cães. In: Resumos do XVI Congresso Brasileiro de Parasitologia, Poços de Caldas, p.57, 1999.

14. Fernandes FF, Silva IG. Eficiência da deltametrina sobre o Aedes (Stegomyia) aegypti (Linnaeus, 1762), aplicada em ultra-baixovolume na dosagem de 1,0g/ha. Entomologia y Vectores 6:581589, 1999.

15. Fernandes FF, Silva IG, Gomes AG, Silva JRV, Silva OR. Ação carrapaticida in vitro de "Butox $\mathrm{P}^{{ }^{\circledR}}$ sobre larvas de Rhipicephalus sanguineus (Latreille, 1806) (Acari, Ixodidae). In: Anais do XV Congresso Brasileiro de Parasitologia, Salvador p. 200, 1997.

16. Fernandes FF, Silva OR, Silva JRV. Estudo da ação de cypermethrin $15 \%$ sobre larvas de Rhipicephalus sanguineus (Latreille, 1806). In: Resumos do XVI Congresso Brasileiro de Entomologia, Rio de Janeiro, p.1076, 1998. 
17. Flechtmann CHW. Ácaros de importância médico-veterinária. Nobel, São Paulo, 1973.

18. Food and Agriculture Organization of The United Nations (FAO). Acaricide resistance test Kit Instructions for use, World Acaricide Resistance Reference Centre (WARRC), Edition 11/1995, Berlin, Germany, 1995.

19. Franc M, Cadiergues MC. Activity of a deltamethrin shampoo against Ctenocephalides felis and Rhipicephalus sanguineus in dogs. Veterinary Parasitology 81:341-346, 1999.

20. Furlong J. Controle do carrapato dos bovinos na Região Sudeste do Brasil. Cadastro Técnico da Escola de Veterinária da Universidade Federal de Minas Gerais 8:49-61, 1993.

21. Gladney WJ, Dawkins CC. The brown dog tick: laboratory tests of acaricides. Southwestern Entomologist 1:184-189, 1976.

22. Hamilton JGC, Papadopoulus E, Harrison SJ, Lloyd CM, Walker AR. Evidence for a mounting sex pheromone in the brown ear tick Rhipicephalus appendiculatus, Neuman 1901 (Acari: Ixodidae). Experimental \& Applied Acarology 18:331-338, 1994.

23. Harrison BA, Engber BR, Apperson CS. Ticks (Acari: Ixodidae) uncommonly found biting humans in North Carolina. Journal of Vector Ecology 22:6-12, 1997.

24. Hervé JJ. El modo de acción de los piretroides y el problema de la resistencia a estos compuestos. In: Nominé G (ed) Deltametrin Monografia. Roussel-Uclaf, Paris, p.67-107, 1983.

25. Hoogstraal $\mathrm{H}$. Ticks in relation to human diseases caused by Rickettsia species. Annual Review of Entomology 12:377-420, 1967.

26. Kaaya GP, Mwangi EN, Ouna EA. Prospects for biological of livestock ticks, Rhipicephalus appendiculatus and Amblyomma variegatum, using the entomogenous fungi Beauveria bassiana and Metarhizium anisopliae. Journal Invertebrate Pathology 67:1520, 1996

27. Lemos ER, Machado RD, Coura JR, Guimaraes MA, Freire NM, Amorim M, Gazeta GS. Epidemiological aspects of the Brazilian spotted fever: seasonal activity of ticks collected in an endemic area in São Paulo, Brazil. Revista da Sociedade Brasileira de Medicina Tropical 30:181-185, 1997.

28. Lemos ER, Machado RD, Pires FD, Machado SL, Costa LM, Coura JR. Rickettsiae-infected ticks in an endemic area of spotted fever in the State of Minas Gerais, Brazil. Memórias do Instituto Oswaldo Cruz 92:477-481, 1997.

29. Lodos J, Ochagavia ME, Rodriguez M, De la Fuente J. A simulation study of the effects of acaricides and vaccination on Boophilus cattle-tick populations. Preventive Veterinary Medicine 38:47-63, 1999.
30. Ministério da Agricultura. Portaria $n^{\circ} 90$ de 04 de dezembro de 1989. Normas para produção e utilização de produtos antiparazitários. Diário Oficial, 22 jan, seção 1, coluna 2, 1990.

31. Nominé G. Deltametrín Monografía. Roussel-Uclaf, Paris, p. 219231, 1993.

32. Polyakov DK, Smirnova OI. (Resistance of ticks to organophosphorus and carbamate acaricides) Rezistentnost iksodid k FOS i karbamatam. Veterinariya, Moscow, USSR (1976) $N^{\circ}$ 5, 66-68. In: Veterinary Bulletin 46:324, 1976.

33. Redondo M, Fragoso H, Ortiz M, Montero C, Lona J, Medellin JA, Fria R, Hernandez V, Franco R, Machado $\mathrm{H}$, Rodriguez $\mathrm{M}$, de la Fuente J. Integrated control of acaricide-resistant Boophilus microplus populations on grazing cattle in Mexico using vaccination with Gavac (TM) and amidine treatments. Experimental and Applied Acarology 23:841-849, 1999.

34. Rodrigues PC. Bioestatística. Editora Universidade Federal Fluminense, Niterói, 1986.

35. Sartor AA, Cunha DW, Daemon E. Aspectos da biologia de Rhipicephalus sanguineus (Latreille, 1806) (Acari: Ixodidae) em condições de laboratório: fase parasitária de larvas, ninfas e fêmeas e não parasitária de larvas e ninfas. Revista Brasileira de Medicina Veterinária 18:14-17, 1996.

36. Sexton DJ, Burgdorfer W, Thomas L, Norment BR. Rocky Mountain spotted fever in Mississippi: survey for spotted fever antibodies in dogs and for spotted fever group Rickettsiae in dog ticks. American Journal Epidemiology 103:192-197, 1976.

37. Silva IG, Camargo MF, Guimarães FL, Elias M, Oliveira AWS. Estudo da eficácia da deltametrina (K-otrine UBV 0,4\% e 1\%) no combate ao Aedes (Stegomyia) aegypti (Linnaeus, 1762) e ao Culex quinquefasciatus. Revista de Patologia Tropical 2:49-56, 1993.

38. Shaw RD. Culture of an organophosphorus resistant strain of Boophilus microplus (Can). Bulletin Entomological Research 56:389-404, 1966.

39. Stone BF, Haydock KP. A method for measuring the acaricide susceptibility of the cattle tick Boophilus microplus (Can). Bulletin Entomological Research 53:563-578, 1962.

40. Tucci EC, Potenza MR, Dall'occo PL, Rodrigues CL. Avaliação de extratos vegetais no controle de Dermanyssus gallinae (Degeer, 1778) (Acari, Dermanyssidae). In: Resumos do XVII Congresso Brasileiro de Entomologia, VIII Encontro Nacional de Fitossanitaristas, Rio de Janeiro, p. 1078, 1998.

41. Veronesi R. Doenças infecciosas e parasitárias, $7^{\text {an }}$ edição. Guanabara Koogan, Rio de Janeiro, 1982.

42. World Health Organization. Doc. TRS/443. World Health Organization, Genéve, 1970. 